\title{
A method of artificial eye manufacture
}

\author{
HELENE JAMES, JEAN R. ELLIS, AND WALLACE S. FOULDS \\ From the Tennent Institute of Ophthalmology, University of Glasgow, and \\ Glasgow Eye Infirmary
}

In most hospitals in the United Kingdom and in many other countries, the provision of an artificial eye depends upon an exchange of information between an artificial eye fitter and an artificial eye manufacturer who may be situated some distance away and who, in most instances, does not see the patient. The centralization of manufacturing facilities may have some advantages and while, in general, the transfer of information between fitter and manufacturer results in an adequate prosthesis, there are inherent difficulties not only in the accurate interpretation of the fitter's instructions but also in relation to the commonly used methods of manufacture. Because of these difficulties, a modified method for the production of artificial eyes has been developed in Glasgow by one of us (HJ) and it is thought that the results obtained by this method justify its being more widely known.

The information transmitted to the manufacturer by a fitter will usually include a wax model of the prosthesis into which a plastic disc has been set to indicate the position of the iris and pupil (Fig. I), together with written instructions about the colour and texture of the iris, the colour of the sclera, and the presence and pattern of vascular markings and other discolourations.

The main bulk of the artificial eye is manufactured to conform with the wax model. A circular hole is drilled into its anterior surface to take a painted plastic disc representing the iris and subsequently a clear acrylic cornea is added anteriorly (lensing) after the addition of vascular and other markings to the sclera.

The problems with this method are first, that the manufacturers are at a great disadvantage in never having seen the patient for whom the eye is being prepared. They must rely upon the interpretation of possibly ambiguous instructions, for qualitative information about the patient's normal eye is difficult to convey in writing. It is upon a combination of many small details that the appearance of an artificial eye depends and a perfect colour match of the iris, for example, can be spoiled completely

Address for reprints: Professor W. S. Foulds, 3 The University, Glasgow Gi2 8QG

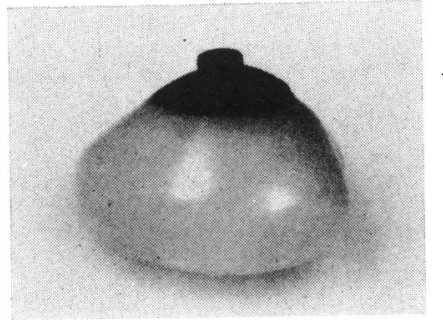

FIG. I Wax model of prosthesis used in conventional artificial eye manufacture with embedded plastic disc which marks position of iris. Central protrusion indicates position of pupil

by too much or too little in the way of vascular markings on the related sclera.

The second problem with the usual method of manufacture is a possible inaccuracy in the position of the iris resulting from difficulty in placing the plastic former accurately on the wax model. The small central protrusion upon the plastic former which marks the position of the pupil may on occasion foul the upper lid margin making accurate centration difficult (Fig. 2). A further inaccuracy may result from slight errors in boring into the half-made prosthesis. An iris misplaced because of this, needs only to be a little

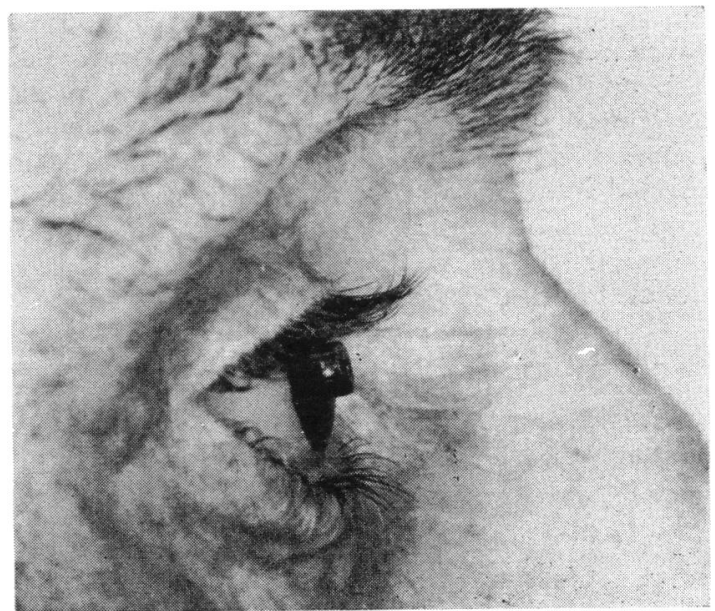

FIG. 2 Conventional wax model in situ. Central protrusion may foul upper lid making centration difficult 


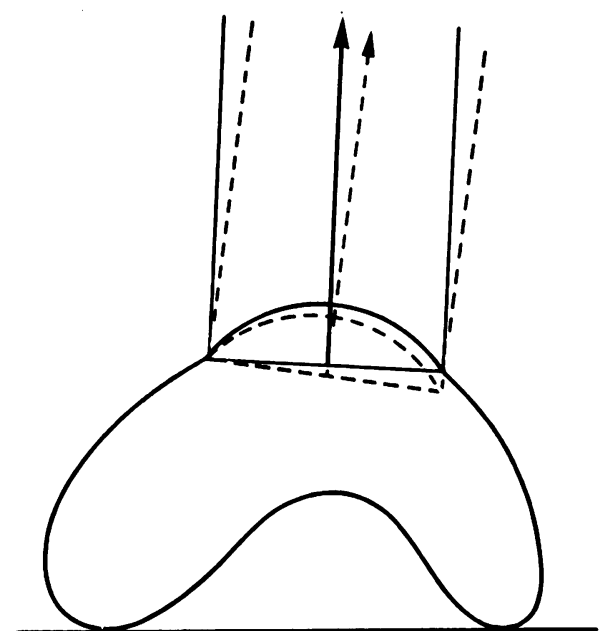

FIG. 3 Diagram to illustrate how slight eccentricity in drilling bed in prosthesis for insertion of iris disc may alter the 'gaze' of artificial eye and necessitate major modification of prosthesis

out of true to require major alteration to the shape of the artificial eye to rectify it (Fig. 3).

In an attempt to circumvent these problems, it was decided to have the artificial eye fitter not only to fit the prosthesis but to manufacture it as well. As most of the materials and techniques used in the manufacture of artificial eyes originate in dentistry, it was decided to adapt the method whereby the bulk of the dentures is added to the teeth, and to try to add the sclera to the iris rather than vice versa. Thus the iris is painted with direct reference to the colour and pattern of the remaining eye and the painted iris disc is 'lensed' with its clear acrylic cornea in a specially constructed flask (Fig. 4) as the first stage in the production of the prosthesis. A mould of the patient's socket is taken in the conventional manner and a wax model of the mould prepared. The combined iris and cornea is then inserted into this wax model in place of the conventional plastic disc used to achieve centration of the pupil and iris, and the position of the iris is modified until it is satisfactory.
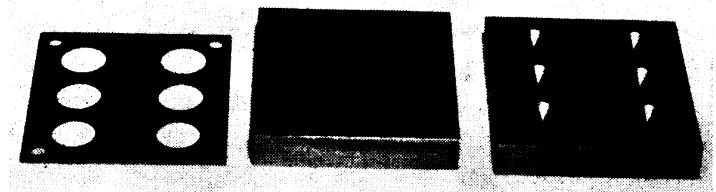

FIG. 4 Special flask used to 'lens' previously painted iris discs. Discs themselves are prepared using same flask substituting flat surfaced base for base with corneal indents illustrated
A small blob or projection of rapidly polymerizing acrylic is added to the apex of the cornea before the wax model with its embedded iris and cornea is inserted into wet plaster-of-Paris in a compress flask. When the plaster has set, the wax is carefully removed leaving the painted iris and cornea held in place by the added projection which is gripped by the plaster (Fig. 5).

The space in the mould previously occupied by the wax is filled with monomer and catylist mixed to match the colour of the fellow sclera and the prosthesis is then heat cured.

The artificial eye complete with iris and cornea is taken from the flask and the projection removed from the cornea. A thin layer of acrylic, mixed to the required sclerotic shade, is spread over the cornea and heat cured. By carefully sandpapering this layer one can reproduce the gentle merging

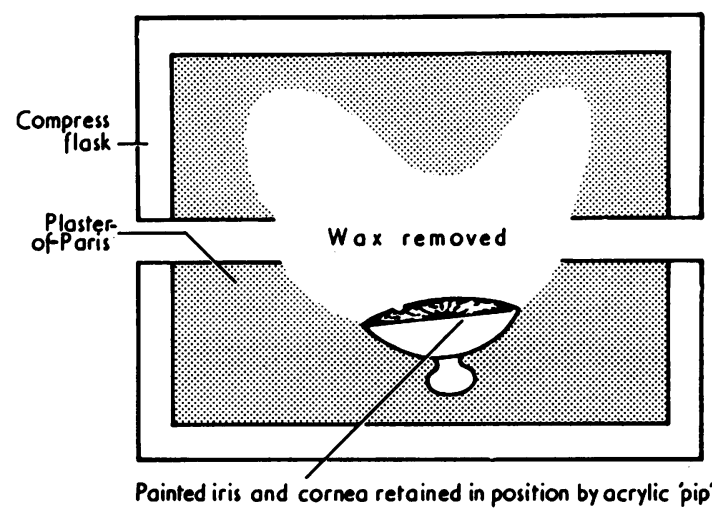

FIG. 5 Diagram of compress flask after removal of wax which forms most of prosthesis. Final iris and cornea are retained in mould by temporary projection of acrylic applied to cornea

of the cornea with the sclera at the limbus seen in the normal eye and eliminate the harsh outline of the limbus which mars so many prostheses. Other effects, such as an arcus senilis or some degree of lens opacity, can also be reproduced at this stage by appropriate removal of the acrylic layer which has been applied over the cornea.

When the appearance of the iris and cornea has been modified to match the patient's remaining eye, the scleral surface is reduced by $\mathrm{I-2} \mathrm{mm}$, and vascular markings and stains on the sclera are added to match those of the fellow eye. At this stage the fitter who suspects that further adjustment to the anterior surface of the prosthesis might be required after a short period of wear, can remove a thicker layer of acrylic from the critical positions; thus alteration to the anterior surface of the finished prosthesis, if required, will not remove any of the vascular markings. 
The layer of sclera thus removed is replaced by a mixture of clear acrylic resin which is polymerized and the eye is then finished by polishing.

In certain cases, for example in patients with microphthalmic eyes, the impression of the socket is translated directly into an acrylic trial shell in the manner of a contact lens fitting. The patient wears this for a week to establish the comfort and accuracy of the fit, and then sufficient acrylic is removed from the anterior surface of the trial shell to enable a painted iris and cornea to be affixed with wax. The appearance of the eye is then checked in the patient's socket. Any necessary modifications to the anterior planes of the prosthesis are executed by the addition of wax or the removal of acrylic. The manufacture then proceeds in the usual manner, with the exception that the wax surrounding the painted iris and cornea is softened by pouring hot water on to the trial shell to enable it to be removed from the compress flask, leaving the iris and cornea in position in the mould.

The advantages of using this method are that the iris colour can be checked by the fitter/maker and also by the patient at an early stage of manufacture. As in all cosmetic effects, personal taste enters into the satisfaction of the wearer and, curiously, some patients prefer their artificial eye to be slightly darker, some slightly lighter, than their own. By inserting the painted iris into the wax model, the patient can have a much closer impression of what the finished eye will look like. It is impossible to obtain the patient's reaction to

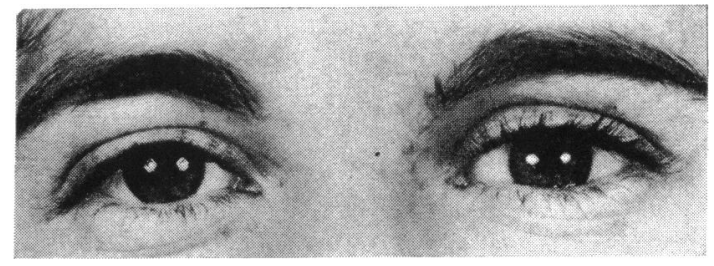

FIG. 6 A satisfactory prosthesis

the appearance of the prosthesis at the fitting stage, when alterations are most easily made, if the conventional blank plastic disc is used in place of the iris.

When the fitter paints and makes the eye, close attention can be paid to the small details which contribute so largely to the final effect, and care can be taken that the vital planes and curves of the shape are not lost by over enthusiastic polishing at the final stage. Certainly the fitter who also makes the eye must have artistic skill and a good colour sense, and he must take total responsibility for the final product. This, however, adds considerably to the satisfaction both from the patient's and fitter's point of view when a successful fitting is achieved (Fig. 6).

We wish to acknowledge the help of $\mathrm{Mr} \mathrm{W}$. Jones, Instrument Technician in the Tennent Institute of Ophthalmology, in relation to the manufacture of the metal flasks. We should also like to thank Mr J. Watt, Chief Technician, and his staff for the photography. 